\title{
Corrigenda
}

Hansjörg Kielhöfer

\section{Critical points of nonconvex and noncoercive functionals}

Received: 25 November 2003 / Accepted: 3 March 2004

Published online: 12 May 2004 - (c) Springer-Verlag 2004

Calc. Var. 16, 243-272 (2003)

The statement (7.1) and the claim after it are not correct. We give a proof for the nontriviality of the singular limits, cf. Theorem 7.3. (Theorem 7.1 is correct, but we cannot apply it to prove $u_{\lambda, 0} \neq 0$, if $u_{\lambda, 0}$ is a singular limit for $\lambda \in\left(\lambda^{1}, \lambda^{2}\right)$.)

Assume that $u_{\lambda, 0} \equiv 0$; i.e., for a sequence $\left(\lambda, u_{\lambda, \varepsilon_{k}}\right) \in C_{n, \varepsilon_{k}}^{+}$with $\varepsilon_{k} \searrow 0$ the sequences

$$
\begin{aligned}
& u_{\lambda, \varepsilon_{k}}^{\prime}=z_{\lambda, \varepsilon_{k}} \rightarrow z_{\lambda, 0} \text { pointwise in }\left[0, \frac{1}{n}\right], \\
& z_{\lambda, 0}=0 \text { almost everywhere, } \\
& u_{\lambda, \varepsilon_{k}} \rightarrow 0 \text { uniformly in }\left[0, \frac{1}{n}\right] \text { as } k \rightarrow \infty,
\end{aligned}
$$

cf. (6.1), (6.2). Furthermore, the sequence $\left(z_{\lambda, \varepsilon_{k}}\right)$ is uniformly bounded, and by the monotonicity (4.7) of $z_{\lambda, \varepsilon_{k}}$ the convergence (1) implies that

$$
u_{\lambda, \varepsilon_{k}}^{\prime} \rightarrow 0 \text { uniformly on each }
$$

$$
\text { compact interval of }\left(0, \frac{1}{n}\right) \text {. }
$$

A matrix $\mathbf{R}=\left(a_{i j}\right)_{i, j=1,2}$ with $a_{i j} \in C\left[0, \frac{1}{n}\right]$ defines for $\mathbf{u}=\left(u_{1}, u_{2}\right) \in$ $W^{2,2}\left(0, \frac{1}{n}\right) \times W^{2,2}\left(0, \frac{1}{n}\right)=W^{2,2}\left(0, \frac{1}{n}\right)^{2}$ via $\mathbf{R u}$ a bounded linear operator from $W^{2,2}\left(0, \frac{1}{n}\right)^{2}$ into $L^{2}\left(0, \frac{1}{n}\right)^{2}$. If $\mathbf{R}_{k}=\left(a_{i j}^{k}\right)$ is a sequence, where $a_{i j}^{k}$ is uniformly bounded and converges pointwise to $a_{i j}^{0}$, then $\mathbf{R}_{k}$ converges to $\mathbf{R}_{0}=\left(a_{i j}^{0}\right)$ in the Banach space of bounded linear operators from $W^{2,2}\left(0, \frac{1}{n}\right)^{2}$ into $L^{2}\left(0, \frac{1}{n}\right)^{2}$, denoted $\mathbf{L}\left(W^{2,2}\left(0, \frac{1}{n}\right)^{2}, L^{2}\left(0, \frac{1}{n}\right)^{2}\right) \equiv \mathbf{L}$. For a proof observe that $W^{2,2}\left(0, \frac{1}{n}\right) \subset$ $C\left[0, \frac{1}{n}\right]$ with continuous embedding. We define for fixed $\lambda \in\left(\lambda^{1}, \lambda^{2}\right)$

$$
\mathbf{R}_{0}=\left(\begin{array}{cc}
0 & 1 \\
0 & -W^{\prime \prime}(\lambda)
\end{array}\right), \quad \mathbf{R}_{0}^{t}=\left(\begin{array}{cc}
0 & 0 \\
1 & -W^{\prime \prime}(\lambda)
\end{array}\right)
$$

Hansjörg Kielhöfer: Universität Augsburg, Institut für Mathematik, Universitätsstrasse 14, 86135 Augsburg, Germany (e-mail: Kielhoefer@math.uni-augsburg.de) 
The transposed matrix $\mathbf{R}_{0}^{t}$ defines the second order system

$$
\begin{aligned}
& \mathbf{v}_{0}^{\prime \prime}+\mu_{0} \mathbf{R}_{0}^{t} \mathbf{v}_{0}=\mathbf{0} \text { in }\left[0, \frac{1}{n}\right], \\
& \mathbf{v}_{0}(0)=\mathbf{v}_{0}\left(\frac{1}{n}\right)=\mathbf{0}, \quad \text { where } \\
& \mu_{0}=-\frac{n^{2} \pi^{2}}{W^{\prime \prime}(\lambda)}>0 \text { since } W^{\prime \prime}(\lambda)<0, \text { see Fig. } 1, \\
& v_{01}(x) \equiv 0, \quad v_{02}(x)=\sin n \pi x .
\end{aligned}
$$

Let $\mathbf{N}=\operatorname{span}\left[\mathbf{v}_{0}\right] \subset L^{2}\left(0, \frac{1}{n}\right)^{2}$ and $\mathbf{N}^{\perp}$ be its orthogonal complement in $L^{2}\left(0, \frac{1}{n}\right)^{2}$. We define

$$
\begin{aligned}
& \mathbf{F}: \mathbb{R} \times\left(W^{2,2}\left(0, \frac{1}{n}\right)^{2} \cap W_{0}^{1,2}\left(0, \frac{1}{n}\right)^{2} \cap \mathbf{N}^{\perp}\right) \times \mathbf{L} \rightarrow L^{2}\left(0, \frac{1}{n}\right)^{2}, \\
& \mathbf{F}(\mu, \mathbf{w}, \mathbf{R})=\left(\mathbf{v}_{0}+\mathbf{w}\right)^{\prime \prime}+\mu \mathbf{R}\left(\mathbf{v}_{0}+\mathbf{w}\right) .
\end{aligned}
$$

Then

(6)

$$
\begin{aligned}
& \mathbf{F}\left(\mu_{0}, \mathbf{0}, \mathbf{R}_{0}^{t}\right)=0, \\
& D_{\mu} \mathbf{F}\left(\mu_{0}, \mathbf{0}, \mathbf{R}_{0}^{t}\right)=\mathbf{R}_{0}^{t} \mathbf{v}_{0}=-\frac{1}{\mu_{0}} \mathbf{v}_{0}^{\prime \prime}, \\
& D_{\mathbf{w}} \mathbf{F}\left(\mu_{0}, \mathbf{0}, \mathbf{R}_{0}^{t}\right) \mathbf{h}=\mathbf{h}^{\prime \prime}+\mu_{0} \mathbf{R}_{0}^{t} \mathbf{h} .
\end{aligned}
$$

Since

$$
\begin{gathered}
h_{2}^{\prime \prime}-\mu_{0} W^{\prime \prime}(\lambda) h_{2}=h_{2}^{\prime \prime}+n^{2} \pi^{2} h_{2}=\sin n \pi x, \\
h_{2}(0)=h_{2}\left(\frac{1}{n}\right)=0,
\end{gathered}
$$

has no solution $h_{2} \in W^{2,2}\left(0, \frac{1}{2}\right) \cap W_{0}^{1,2}\left(0, \frac{1}{n}\right)$, the element $\mathbf{R}_{0}^{t} \mathbf{v}_{0}$ is not in the range of $D_{\mathbf{w}} \mathbf{F}\left(\mu_{0}, \mathbf{0}, \mathbf{R}_{0}^{t}\right)$. By the Fredholm property of $D_{\mathbf{w}} \mathbf{F}\left(\mu_{0}, \mathbf{0}, \mathbf{R}_{0}^{t}\right)$ this implies that

$$
D_{(\mu, \mathbf{w})} \mathbf{F}\left(\mu_{0}, \mathbf{0}, \mathbf{R}_{0}^{t}\right):
$$

$$
\mathbb{R} \times\left(W^{2,2}\left(0, \frac{1}{n}\right)^{2} \cap W_{0}^{1,2}\left(0, \frac{1}{n}\right)^{2} \cap \mathbf{N}^{\perp}\right) \rightarrow L^{2}\left(0, \frac{1}{n}\right)^{2}
$$

is an isomorphism.

The Implicit Function Theorem then yields a unique solution $(\mu, \mathbf{w})$ of $\mathbf{F}(\mu, \mathbf{w}, \mathbf{R})=\mathbf{0}$ near $\left(\mu_{0}, \mathbf{0}\right)$ for any $\mathbf{R}$ near $\mathbf{R}_{0}^{t}$ in $\mathbf{L}$.

Define

$$
\mathbf{R}_{\varepsilon_{k}}=\left(\begin{array}{cc}
0 & 1 \\
\varepsilon_{k} g\left(u_{\lambda, \varepsilon_{k}}\right) & -W^{\prime \prime}\left(\lambda+u_{\lambda, \varepsilon_{k}}^{\prime}\right)
\end{array}\right),
$$


where $g$ is given in (7.1). By assumptions (1), (2),

$$
\mathbf{R}_{\varepsilon_{k}} \rightarrow \mathbf{R}_{0} \text { in } \mathbf{L} \text { as } k \rightarrow \infty
$$

and the considerations before imply the existence of $\left(\mu_{\varepsilon_{k}}, \mathbf{w}_{\varepsilon_{k}}\right)$ near $\left(\mu_{0}, \mathbf{0}\right)$ such that $\mathbf{v}_{\varepsilon_{k}}=\mathbf{v}_{0}+\mathbf{w}_{\varepsilon_{k}}$ satisfies

$$
\begin{aligned}
& \mathbf{v}_{\varepsilon_{k}}^{\prime \prime}+\mu_{\varepsilon_{k}} \mathbf{R}_{\varepsilon_{k}}^{t} \mathbf{v}_{\varepsilon_{k}}=\mathbf{0} \text { in }\left[0, \frac{1}{n}\right] \\
& \mathbf{v}_{\varepsilon_{k}}(0)=\mathbf{v}_{\varepsilon_{k}}\left(\frac{1}{n}\right)=\mathbf{0} .
\end{aligned}
$$

Since the second component $v_{\varepsilon_{k}, 2}$ of $\mathbf{v}_{\varepsilon_{k}}$ is near $v_{02}(x)=\sin n \pi x$ in the topology of $W^{2,2}\left(0, \frac{1}{n}\right) \subset C^{1}\left[0, \frac{1}{n}\right]$, that component $v_{\varepsilon_{k}, 2}$ is positive $\left(0, \frac{1}{n}\right)$. The first equation of (11) gives in view of the positivity of $g\left(u_{\lambda, \varepsilon_{k}}\right)$, cf. (7.1),

$$
v_{\varepsilon_{k}, 1}^{\prime \prime}=-\mu_{\varepsilon_{k}} \varepsilon_{k} g\left(u_{\lambda, \varepsilon_{k}}\right) v_{\varepsilon_{k}, 2}<0 \quad \text { in }\left(0, \frac{1}{n}\right),
$$

such that the first component $v_{\varepsilon_{k}, 1}$ of $\mathbf{v}_{\varepsilon_{k}}$ is positive in $\left(0, \frac{1}{n}\right)$, too.

By the Euler-Lagrange equation (2.4) we have also the following identity:

$$
\begin{gathered}
\mathbf{u}_{\varepsilon_{k}}^{\prime \prime}+\frac{1}{\varepsilon_{k}} \mathbf{R}_{\varepsilon_{k}} \mathbf{u}_{\varepsilon_{k}}=\mathbf{0} \text { in }\left[0, \frac{1}{n}\right], \\
\mathbf{u}_{\varepsilon_{k}}(0)=\mathbf{u}_{\varepsilon_{k}}\left(\frac{1}{n}\right)=\mathbf{0}, \text { where } \\
\mathbf{u}_{\varepsilon_{k}}=\left(u_{\lambda, \varepsilon_{k}},-\varepsilon_{k} u_{\lambda, \varepsilon_{k}}^{\prime \prime}\right) .
\end{gathered}
$$

By (4.2) both components of $\mathbf{u}_{\varepsilon_{k}}$ are positive in $\left(0, \frac{1}{n}\right)$.

By its construction via the Implicit Function Theorem the solution $\mathbf{v}_{\varepsilon_{k}}$ of (11) is near $\mathbf{v}_{0}$ in the topology of $W^{2,2}\left(0, \frac{1}{n}\right) \subset C^{1}\left[0, \frac{1}{n}\right]$. Therefore (1), (2), and the second equation of (11) imply that

$$
v_{\varepsilon_{k}, 2} \text { is near } v_{02}(x)=\sin n \pi x
$$$$
\text { in } C^{1}\left[0, \frac{1}{n}\right] \text { and in } C^{2}\left[\delta, \frac{1}{n}-\delta\right]
$$

for any $\delta>0$, provided that $k \geq k(\delta)$. 
This, in turn, has the following consequences:

$$
\begin{array}{ll}
v_{\varepsilon_{k}, 2}^{\prime \prime}(x)<0 & \text { for all } x \in\left[\delta, \frac{1}{n}-\delta\right], \\
v_{\varepsilon_{k}, 2}^{\prime}\left(x_{k}\right)=0 & \text { for a unique } x_{k} \in\left(\delta, \frac{1}{n}-\delta\right) \\
v_{\varepsilon_{k}, 2}^{\prime}(x) \geq 0 & \text { for } x \in\left[\delta, x_{k}\right], \\
v_{\varepsilon_{k}, 2}^{\prime}(x) \leq 0 & \text { for } x \in\left[x_{k}, \frac{1}{n}-\delta\right], \\
\text { for all } k \geq k_{0}(\delta) .
\end{array}
$$

We choose a cut-off function $\eta_{k} \in C^{2}\left[0, \frac{1}{n}\right]$ having the following properties $\left(\delta>0\right.$ is fixed and $\left.k \geq k_{0}(\delta)\right)$ :

$$
\begin{aligned}
& \eta_{k}(x) \geq 0 \text { for all } x \in\left[0, \frac{1}{n}\right], \\
& \operatorname{supp}\left(\eta_{k}\right) \subset\left[\delta, \frac{1}{n}-\delta\right], \eta_{k}(x)>0 \text { for } x \in\left(\delta, \frac{1}{n}-\delta\right), \\
& v_{\varepsilon_{k}, 2}^{\prime}(x) \eta_{k}^{\prime}(x) \geq 0 \text { for all } x \in\left[0, \frac{1}{n}\right], \\
& \eta_{k}^{\prime \prime}(x)+C_{0} \eta_{k}(x) \geq 0 \text { for all } x \in\left[0, \frac{1}{n}\right],
\end{aligned}
$$

and for some $C_{0}>n^{2} \pi^{2}$.

Next we choose $k \geq k_{0}(\delta)$ so large such that for fixed $\lambda \in\left(\lambda^{1}, \lambda^{2}\right)$ where $W^{\prime \prime}(\lambda)<0$ the following holds true:

$$
\begin{aligned}
& W^{\prime \prime}\left(\lambda+u_{\lambda, \varepsilon_{k}}^{\prime}\right) \leq-c_{0}<0 \text { on }\left[\delta, \frac{1}{n}-\delta\right], \text { cf. }(2), \\
& \frac{1}{2 \varepsilon_{k}}\left(-W^{\prime \prime}\left(\lambda+u_{\lambda, \varepsilon_{k}}^{\prime}\right)-\mu_{\varepsilon_{k}} \frac{v_{1, \varepsilon_{k}}}{v_{2, \varepsilon_{k}}}\right) \geq C_{0} \text { on }\left[\delta, \frac{1}{n}-\delta\right], \\
& \varepsilon_{k} \mu_{\varepsilon_{k}}<\frac{1}{2},
\end{aligned}
$$

which is possible, since $\mu_{\varepsilon_{k}} \rightarrow \mu_{0}>0, v_{2, \varepsilon_{k}} \geq c_{1}>0$, and $v_{1, \varepsilon_{k}} \rightarrow 0$ uniformly on $\left[\delta, \frac{1}{n}-\delta\right]$ as $k \rightarrow \infty$.

With the abbreviations $\mathbf{v}_{\varepsilon_{k}}=\left(v_{\varepsilon_{k}, 1}, v_{\varepsilon_{k}, 2}\right)=\left(v_{1}, v_{2}\right), \mathbf{u}_{\varepsilon_{k}}=\left(u_{1}, u_{2}\right)=$ $\left(u,-\varepsilon u^{\prime \prime}\right), \eta_{k}=\eta, \mu_{\varepsilon_{k}}=\mu, \varepsilon_{k}=\varepsilon$, we obtain from (11) and (13), where 
$(\quad, \quad)_{0,2}=(\quad, \quad)$ denotes the scalar product in $L^{2}\left(0, \frac{1}{n}\right)$ :

$$
\begin{aligned}
& \left(u_{2},\left(v_{2} \eta\right)^{\prime \prime}\right)_{0,2} \\
& =\left(u_{2}, v_{2}^{\prime \prime} \eta\right)+2\left(u_{2}, v_{2}^{\prime} \eta^{\prime}\right)+\left(u_{2}, v_{2} \eta^{\prime \prime}\right) \\
& =-\mu\left(u_{2}, v_{1} \eta\right)+\mu\left(u_{2}, W^{\prime \prime}\left(\lambda+u^{\prime}\right) v_{2} \eta\right)+2\left(u_{2}, v_{2}^{\prime} \eta^{\prime}\right)+\left(u_{2}, v_{2} \eta^{\prime \prime}\right) \\
& =\left(u_{2}^{\prime \prime}, v_{2} \eta\right)_{0,2} \\
& =-\frac{1}{\varepsilon}\left(\varepsilon g(u) u_{1}, v_{2} \eta\right)+\frac{1}{\varepsilon}\left(W^{\prime \prime}\left(\lambda+u^{\prime}\right) u_{2}, v_{2} \eta\right) \\
& =-\left(g(u) u, v_{2} \eta\right)-\left(W^{\prime \prime}\left(\lambda+u^{\prime}\right) u^{\prime \prime}, v_{2} \eta\right)
\end{aligned}
$$

Inserting $u_{2}=-\varepsilon u^{\prime \prime}$ into the third line of (18) we finally get:

$$
\begin{aligned}
0= & (1-\varepsilon \mu)\left(W^{\prime \prime}\left(\lambda+u^{\prime}\right) u^{\prime \prime}, v_{2} \eta\right)+\left(g(u) u, v_{2} \eta\right) \\
& +\varepsilon \mu\left(u^{\prime \prime}, v_{1} \eta\right)-2 \varepsilon\left(u^{\prime \prime}, v_{2}^{\prime} \eta^{\prime}\right)-\varepsilon\left(u^{\prime \prime}, v_{2} \eta^{\prime \prime}\right) \text { or } \\
0= & \left(\frac{1}{2}-\varepsilon \mu\right)\left(W^{\prime \prime}\left(\lambda+u^{\prime}\right) u^{\prime \prime}, v_{2} \eta\right)+\left(g(u) u, v_{2} \eta\right) \\
& +\varepsilon\left((-u)^{\prime \prime} v_{2}, \eta^{\prime \prime}+\frac{1}{2 \varepsilon}\left(-W^{\prime \prime}\left(\lambda+u^{\prime}\right)-\mu \frac{v_{1}}{v_{2}}\right) \eta\right) \\
& +2 \varepsilon\left(\left(-u^{\prime \prime}\right), v_{2}^{\prime} \eta^{\prime}\right) .
\end{aligned}
$$

By the positivity of $\left(-u^{\prime \prime}\right), g(u) u$, (cf. (7.1)), $v_{2}$ in $\left(0, \frac{1}{n}\right)$, the choice of $\eta$ and of $k$ according to (16) $)_{2}$ and (17) 1 implies that the first two summands in the third line of (19) are positive. The property $(17)_{2}$ together with $(16)_{4}$ guarantees that the third summand in the fourth line of (19) is nonnegative. Finally, property $(16)_{3}$ makes the last summand of (19) nonnegative, too, such that the sum of all four terms has to be positive, contradicting (19).

For convenience we include a second proof for the nontriviality of the singular limits. This proof is due to $\mathrm{S}$. Krömer.

We assume that for a sequence $\left(\lambda, u_{\lambda, \varepsilon_{k}}\right) \in C_{n, \varepsilon_{k}}^{+}$with $\varepsilon_{k} \searrow 0$

$$
u_{\lambda, \varepsilon_{k}} \rightarrow 0 \quad \text { in } W^{1,1}\left(0, \frac{1}{n}\right) \text { as } k \rightarrow \infty
$$

which is a consequence of (1), cf. (6.2). With the abbreviations

$$
w=w_{\lambda, \varepsilon_{k}}=\frac{u_{\lambda, \varepsilon_{k}}}{\left\|u_{\lambda, \varepsilon_{k}}\right\|_{1,1}}, \quad u=u_{\lambda, \varepsilon_{k}}, \quad \varepsilon=\varepsilon_{k}
$$


we obtain from the weak form (6.4) of the Euler-Lagrange equation

$$
\begin{gathered}
\int_{0}^{1 / n} \varepsilon_{k} u_{\lambda, \varepsilon_{k}} \varphi^{(4)}+W^{\prime}\left(\lambda+u_{\lambda, \varepsilon_{k}}^{\prime}\right) \varphi^{\prime}+G^{\prime}\left(u_{\lambda, \varepsilon_{k}}\right) \varphi d x=0 \\
\Rightarrow \int_{0}^{1 / n} \varepsilon w \varphi^{(4)}+\left(\frac{W^{\prime}\left(\lambda+u^{\prime}\right)-W^{\prime}(\lambda)}{u^{\prime}}-W^{\prime \prime}(\lambda)\right) w^{\prime} \varphi^{\prime} d x \\
\quad=\int_{0}^{1 / n}\left(-W^{\prime \prime}(\lambda)\right) w^{\prime} \varphi^{\prime}+g(u) w \varphi d x, \text { cf. }(7.1), \\
\quad \text { for all test functions } \varphi \in C_{0}^{\infty}\left(0, \frac{1}{n}\right) .
\end{gathered}
$$

Since $\left\|w_{\lambda, \varepsilon_{k}}\right\|_{0,1} \leq 1$, the first summand converges to zero as $k \rightarrow \infty$. Furthermore, since $u^{\prime}=u_{\lambda, \varepsilon_{k}}^{\prime}$ is uniformly bounded by (5.1),

$$
\begin{aligned}
& \left|\int_{0}^{1 / n}\left(\frac{W^{\prime}\left(\lambda+u^{\prime}\right)-W^{\prime}(\lambda)}{u^{\prime}}-W^{\prime \prime}(\lambda)\right) w^{\prime} \varphi^{\prime} d x\right| \\
& \leq C_{1} \int_{0}^{1 / n}\left|u^{\prime}\left\|w^{\prime}\right\| \varphi^{\prime}\right| d x=C_{1} \int_{\delta}^{1 / n-\delta}\left|u^{\prime}\left\|w^{\prime}\right\| \varphi^{\prime}\right| d x \\
& \text { if } \operatorname{supp}(\varphi) \subset\left(\delta, \frac{1}{n}-\delta\right) \\
& \leq C_{2}\left\|u_{\lambda, \varepsilon_{k}}^{\prime}\right\|_{C\left[\delta, \frac{1}{n}-\delta\right]}\left\|w_{\lambda, \varepsilon_{k}}\right\|_{1,1} \rightarrow 0 \text { as } k \rightarrow \infty
\end{aligned}
$$

by the convergence (2) and by definition (21). Since the sequence $\left(w_{\lambda, \varepsilon_{k}}\right)$ is bounded in $W^{1,1}\left(0, \frac{1}{n}\right)$, the compact embedding of $W^{1,1}\left(0, \frac{1}{n}\right)$ into $L^{1}\left(0, \frac{1}{n}\right)$ implies the convergence of a subsequence to some $w_{\lambda, 0}$ in $L^{1}\left(0, \frac{1}{n}\right)$. Without changing the notation,

$$
\begin{aligned}
& w_{\lambda, \varepsilon_{k}} \rightarrow w_{\lambda, 0} \text { in } L^{1}\left(0, \frac{1}{n}\right) \text { as } k \rightarrow \infty, \\
& w_{\lambda, 0} \geq 0 \text { a.e. in }\left(0, \frac{1}{n}\right)
\end{aligned}
$$

since $w_{\lambda, \varepsilon_{k}}>0$ in $\left(0, \frac{1}{n}\right)$, cf. (4.2). We claim that for another subsequence

$$
w_{\lambda, \varepsilon_{k}}^{\prime} \rightarrow \tilde{w}_{\lambda, 0} \quad \text { pointwise in }\left(0, \frac{1}{n}\right) .
$$

Indeed, since $\left\|w_{\lambda, \varepsilon_{k}}^{\prime}\right\|_{0,1} \leq 1$ and $w_{\lambda, \varepsilon_{k}}^{\prime}$ is monotonically decreasing in $\left[0, \frac{1}{n}\right]$, we have

$$
\begin{aligned}
I_{N} & =\left\{x \in\left[0, \frac{1}{n}\right]|| w_{\lambda, \varepsilon_{k}}^{\prime}(x) \mid \leq N \text { for all } k\right\} \\
& \supset\left[\frac{1}{N}, \frac{1}{n}-\frac{1}{N}\right] \text { for } N>2 n .
\end{aligned}
$$


Assume that $w_{\lambda, \varepsilon_{k}}^{\prime}\left(x_{N}\right)>N$ or $w_{\lambda, \varepsilon_{k}}^{\prime}\left(x_{N}\right)<-N$ for some $k$ and some $x_{N} \in\left[\frac{1}{N}, \frac{1}{n}-\frac{1}{N}\right]$. Then

$$
\begin{aligned}
& w_{\lambda, \varepsilon_{k}}^{\prime}(x)>N \text { for all } x \in\left[0, \frac{1}{N}\right] \text { or } \\
& w_{\lambda, \varepsilon_{k}}^{\prime}(x)<N \text { for all } x \in\left[\frac{1}{N}, \frac{1}{n}-\frac{1}{N}\right], \text { whence } \\
& \left\|w_{\lambda, \varepsilon_{k}}^{\prime}\right\|_{0,1}>1 \text { in both cases. }
\end{aligned}
$$

This contradiction proves $\left\|w_{\lambda, \varepsilon_{k}}^{\prime}\right\|_{C\left[\frac{1}{N}, \frac{1}{n}-\frac{1}{N}\right]} \leq N$ for all $k$, and Helly's Theorem implies the pointwise convergence of a subsequence to a monotonically decreasing function that is bounded on $\left[\frac{1}{N}, \frac{1}{n}-\frac{1}{N}\right]$. Since the (countable) union of all intervals $\left[\frac{1}{N}, \frac{1}{n}-\frac{1}{N}\right]$ is $\left(0, \frac{1}{n}\right)$, the diagonal of all respective subsequences fulfills (25), and moreover

$$
\left|\tilde{w}_{\lambda, 0}(x)\right| \leq N \text { for } x \in\left[\frac{1}{N}, \frac{1}{n}-\frac{1}{N}\right] \text { and all } N>2 n,
$$

$$
\text { and } \tilde{w}_{\lambda, 0} \text { is monotonically decreasing in }\left(0, \frac{1}{n}\right) \text {. }
$$

Finally, properties (25), (26), (28) imply that

$$
w_{\lambda, \varepsilon_{k}}^{\prime} \rightarrow \tilde{w}_{\lambda, 0} \quad \text { in } L_{l o c}^{1}\left(0, \frac{1}{n}\right)
$$

which means by the definition of a weak derivative that the limit given in (24) has the following properties:

$$
\begin{aligned}
& w_{\lambda, 0} \in W_{l o c}^{1,1}\left(0, \frac{1}{n}\right), w_{\lambda, 0}^{\prime}=\tilde{w}_{\lambda, 0}, \text { and } \\
& w_{\lambda, \varepsilon_{k}} \rightarrow w_{\lambda, 0} \text { in } W_{l o c}^{1,1}\left(0, \frac{1}{n}\right) .
\end{aligned}
$$

Inserting the subsequence satisfying (30) into (22), we obtain by (23) in the limit (cf. (1) 3 )

$$
\begin{aligned}
& \int_{0}^{1 / n}\left(-W^{\prime \prime}(\lambda)\right) w_{\lambda, 0}^{\prime} \varphi^{\prime}+g(0) w_{\lambda, 0} \varphi d x=0 \\
& \text { for all } \varphi \in C_{0}^{\infty}\left(0, \frac{1}{n}\right) .
\end{aligned}
$$

By (30) the function $w_{\lambda, 0}$ is in $C\left(0, \frac{1}{n}\right)$, whence by the fundamental lemma of the calculus of variations,

$$
\begin{aligned}
& w_{\lambda, 0} \in C^{2}\left(0, \frac{1}{n}\right) \text { and } \\
& \left.W^{\prime \prime}(\lambda)\right) w_{\lambda, 0}^{\prime \prime}+g(0) w_{\lambda, 0}=0 \text { in }\left(0, \frac{1}{n}\right) .
\end{aligned}
$$


Now, by $(24)_{2}$ and $(28)_{2}$, we have $w_{\lambda, 0} \geq 0$ and $w_{\lambda, 0}^{\prime \prime} \leq 0$ on $\left(0, \frac{1}{n}\right)$.

Since $\lambda \in\left(\lambda^{1}, \lambda^{2}\right)$ where $W^{\prime \prime}(\lambda)<0$ and $g(0)>0$, equation (32) is only satisfied if

$$
\begin{aligned}
& w_{\lambda, 0}=0 \quad \text { on }\left(0, \frac{1}{n}\right) \text { or, by }(24), \\
& w_{\lambda, \varepsilon_{k}} \rightarrow 0 \text { in } L^{1}\left(0, \frac{1}{n}\right) \text { as } k \rightarrow \infty .
\end{aligned}
$$

By the monotonicity (4.7) of $u_{\lambda, \varepsilon_{k}}^{\prime}=z_{\lambda, \varepsilon_{k}}$ there is a unique $x_{k} \in\left(0, \frac{1}{n}\right)$ where $u_{\lambda, \varepsilon_{k}}^{\prime}\left(x_{k}\right)=0$. The boundary conditions $u_{\lambda, \varepsilon_{k}}(0)=u_{\lambda, \varepsilon_{k}}\left(\frac{1}{n}\right)=0$, the positivity of $u_{\lambda, \varepsilon_{k}}$, and $u_{\lambda, \varepsilon_{k}}\left(x_{k}\right)=\max _{x \in\left[0, \frac{1}{n}\right]} u_{\lambda, \varepsilon_{k}}(x)=\left\|u_{\lambda, \varepsilon_{k}}\right\|_{0}$ imply

$$
\begin{array}{r}
\int_{0}^{1 / n}\left|u_{\lambda, \varepsilon_{k}}^{\prime}\right| d x=\int_{0}^{x_{k}} u_{\lambda, \varepsilon_{k}}^{\prime} d x-\int_{x_{k}}^{1 / n} u_{\lambda, \varepsilon_{k}}^{\prime} d x \\
=2 u_{\lambda, \varepsilon_{k}}\left(x_{k}\right)=2\left\|u_{\lambda, \varepsilon_{k}}\right\|_{0} .
\end{array}
$$

The property $u_{\lambda, \varepsilon_{k}}^{\prime \prime}<0$, cf. (4.2), means that $u_{\lambda, \varepsilon_{k}}$ is concave and therefore

$$
\left\|u_{\lambda, \varepsilon_{k}}\right\|_{0,1}=\int_{0}^{1 / n} u_{\lambda, \varepsilon_{k}} d x \geq \frac{1}{2 n}\left\|u_{\lambda, \varepsilon_{k}}\right\|_{0} .
$$

Combining (34) and (35) we obtain

$$
\begin{aligned}
\left\|u_{\lambda, \varepsilon_{k}}^{\prime}\right\|_{0,1} & \leq 4 n\left\|u_{\lambda, \varepsilon_{k}}\right\|_{0,1}, \text { whence } \\
\left\|u_{\lambda, \varepsilon_{k}}\right\|_{1,1} & \leq(4 n+1)\left\|u_{\lambda, \varepsilon_{k}}\right\|_{0,1}, \text { whence } \\
\left\|w_{\lambda, \varepsilon_{k}}\right\|_{0,1} & \geq \frac{1}{4 n+1} \text { for all } k,
\end{aligned}
$$

contradicting (33).

\section{References}

Kielhöfer, H.: Critical points of nonconvex and noncoercive functionals. Calc. Var. 16, 243272 (2003) 